\title{
Searching the QCD critical endpoint with lattice simulations
}

\author{
Szabolcs Borsanyi ${ }^{1}$, Zoltan Fodor ${ }^{1,2,3,4}$, Matteo Giordano ${ }^{2}$, Jana N. Guenther ${ }^{1,5, \star}$, Kornél Kapás ${ }^{2}$, \\ Sandor K. Katz ${ }^{2}$, Kalman K. Szabó ${ }^{1,3}$, Attila Pasztor ${ }^{2}$, Israel Portillo ${ }^{6}$, and Claudia Ratti ${ }^{6}$ \\ ${ }^{1}$ University of Wuppertal, Department of Physics, Wuppertal D-42097, Germany \\ ${ }^{2}$ Eötvös University, Budapest 1117, Hungary \\ ${ }^{3}$ Jülich Supercomputing Centre, Jülich D-52425, Germany \\ ${ }^{4}$ Physics Department, UCSD, San Diego, CA 92093, USA \\ ${ }^{5}$ University of Regensburg, Department of Physics, Regensburg D-93053, Germany \\ ${ }^{6}$ Department of Physics, University of Houston, Houston, TX 77204, USA
}

\begin{abstract}
We discuss the usefulness of various lattice observables especially fluctuations to locate the QCD critical endpoint. We apply different models to interpret our results for the baryon fluctuations up to $\mu^{8}$ from simulations at imaginary chemical potentials.
\end{abstract}

\section{Introduction}

When investigating Quantum Chromodynamics (QCD) an important but challenging goal is the study of the phase diagram. At zero chemical potential lattice QCD predicts a smooth crossover between hadrons and the quark gluon plasma [1-5], taking place in the temperature range $T \simeq 145-165 \mathrm{MeV}$. Due to the sign problem lattice QCD is unable to study the region with finite chemical potential.

With the advent of the second Beam Energy Scan (BESII) at the Relativistic Heavy Ion Collider (RHIC), scheduled for 2019-2020, there is a renewed interest in the heavy ion community towards the phases of QCD at moderate-to-large densities. A rich theoretical effort is being developed in support of the experimental program; several observables are being calculated, in order to constrain the existence and location of the QCD critical point and to observe it experimentally.

Fluctuations of conserved charges (electric charge $Q$, baryon number $B$ and strangeness $S$ ) are important observables for the finite-density investigations. One possible way to extend lattice results to finite density is to perform Taylor expansions of the thermodynamic observables around chemical potential $\mu_{B}=0$ [6-10]: fluctuations of conserved charges are directly related to the Taylor expansion coefficients of such observables. They allow for a comparison between theoretical and experimental results to extract the chemical freeze-out temperature $T_{f}$ and chemical potential $\mu_{B f}$ as functions of the collision energy [11-14]. The higher order fluctuations are also an important signature for the critical endpoint, as they give access to the correlation length $[8,15,16]$.

In this work we apply the method of analytical continuation from imaginary chemical potential [17-21]. It agrees well with the results of the Taylor expansion as shown for the transition temperature [22]. We present results for $\chi_{2}^{B}$ to $\chi_{6}^{B}$ in the temperature range $140 \mathrm{MeV} \leq T \leq 220 \mathrm{MeV}$. Several diagonal and non-diagonal fluctuations of conserved charges up to sixth-order are available in our recent paper [23].

\footnotetext{
^e-mail: Jana.Guenther@t-online.de
} 


\section{Fluctuations}

We present results of an high-precision analysis on a $48^{3} \times 12$ lattice. A more detailed description as well as precise information on the lattice set-up can be found in refs. [23, 24]. We use analytical continuation from imaginary chemical potential to determine the $\chi^{B}$ fluctuations at $\mu_{B}=0$. We analyze data for eight different values of $\mu_{B}=\mathrm{i} \frac{j \pi}{8}$ with $j \in\{0,1,2,3,4,5,6,7\}$. In our analysis we use the following ansatz for the pressure:

$$
\chi_{0}^{B}\left(\hat{\mu}_{B}\right)=\frac{p}{T^{4}}=c_{0}+c_{2} \hat{\mu}_{B}^{2}+c_{4} \hat{\mu}_{B}^{4}+c_{6} \hat{\mu}_{B}^{6}+\frac{4 !}{8 !} c_{4} \epsilon_{1} \hat{\mu}_{B}^{8}+\frac{4 !}{10 !} c_{4} \epsilon_{2} \hat{\mu}_{B}^{10},
$$

where $\epsilon_{1}$ and $\epsilon_{2}$ are drawn randomly from a normal distribution with $\mu=-1.25$ and $\sigma=2.75$. The values were chosen in a way to allow for $\chi_{8}^{B}$ to take the value predicted by the hadron resonance gas, as well as the result from the toy model introduced in section 4 . From the ansatz we can calculate the derivatives that can be measured on the lattice:

$$
\begin{aligned}
& \chi_{1}^{B}\left(\hat{\mu}_{B}\right)=2 c_{2} \hat{\mu}_{B}+4 c_{4} \hat{\mu}_{B}^{3}+6 c_{6} \hat{\mu}_{B}^{5}+\frac{4 !}{7 !} c_{4} \epsilon_{1} \hat{\mu}_{B}^{7}+\frac{4 !}{9 !} c_{4} \epsilon_{2} \hat{\mu}_{B}^{9}, \\
& \chi_{2}^{B}\left(\hat{\mu}_{B}\right)=2 c_{2}+12 c_{4} \hat{\mu}_{B}^{2}+30 c_{6} \hat{\mu}_{B}^{4}+\frac{4 !}{6 !} c_{4} \epsilon_{1} \hat{\mu}_{B}^{6}+\frac{4 !}{8 !} c_{4} \epsilon_{2} \hat{\mu}_{B}^{8}, \\
& \chi_{3}^{B}\left(\hat{\mu}_{B}\right)=24 c_{4} \hat{\mu}_{B}+120 c_{6} \hat{\mu}_{B}^{3}+\frac{4 !}{5 !} c_{4} \epsilon_{1} \hat{\mu}_{B}^{5}+\frac{4 !}{7 !} c_{4} \epsilon_{2} \hat{\mu}_{B}^{7}, \\
& \chi_{4}^{B}\left(\hat{\mu}_{B}\right)=24 c_{4}+360 c_{6} \hat{\mu}_{B}^{2}+c_{4} \epsilon_{1} \hat{\mu}_{B}^{4}+\frac{4 !}{6 !} c_{4} \epsilon_{2} \hat{\mu}_{B}^{6} .
\end{aligned}
$$

We perform a correlated fit for $\chi_{1}^{B}\left(\hat{\mu}_{B}\right), \chi_{2}^{B}\left(\hat{\mu}_{B}\right), \chi_{3}^{B}\left(\hat{\mu}_{B}\right)$ and $\chi_{4}^{B}\left(\hat{\mu}_{B}\right)$ for the different values of $\mu_{B}$ to determine the fitting parameters $c_{2}, c_{4}$ and $c_{6}$. From the parameters we can determine $\chi_{2}^{B}(0)=2 c_{2}$, $\chi_{4}^{B}(0)=24 c_{4}, \chi_{6}^{B}(0)=720 c_{6}$ and $\chi_{8}^{B}(0)=24 c_{4} \epsilon_{1}$. The results are shown in figure 1 . These equations show the relation between $\chi_{4}^{B}$ and $\chi_{8}^{B}$ that are just related by the factor of $\epsilon_{1}$ (in the same way $\chi_{4}^{B}$ and $\chi_{10}^{B}$ are related by a factor of $\epsilon_{2}$ ). In this way we take into account the influence of higher order corrections to our fit function. We choose 1000 different values for $\epsilon_{1}$ and $\epsilon_{2}$ and in addition we include either seven or eight different values of $\mu_{B}$ in our data. All resulting fits are combined in a histogram and weighted with the Akaike information criteria [25], thus allowing to estimate the systematic error. The statistical error is determined by the Jackknife method and both errors are added quadratically to get the combined error shown in the plots.

\section{Cumulants}

For a comparison with heavy ion collision experiments the cumulants of the net baryon distribution are a useful tool. The first four cumulants are the mean $M_{B}$, the variance $\sigma_{B}^{2}$, the skewness $S_{B}$ and the kurtosis $\kappa_{B}$. By forming appropriate ratios, we can cancel out explicit volume factors. However the measured distributions themselves may still depend on the volume, which one should take into account, when comparing to experiments.

Heavy ion collisions with lead or gold take place at $\mu_{B}>0,\left\langle n_{S}\right\rangle=0$ and $\left\langle n_{Q}\right\rangle=0.4\left\langle n_{B}\right\rangle$. Since our simulations are done at $\mu_{S}=\mu_{Q}=0$ and $\mu_{B} \neq 0$ we have to do some calculations to arrive at the same observables that are measured in experiments (see for example [27]). We investigate three different ratios of cumulants and write each as a Taylor expansion:

$$
\frac{M_{B}}{\sigma_{B}^{2}}=\frac{\chi_{1}^{B}\left(T, \hat{\mu}_{B}\right)}{\chi_{2}^{B}\left(T, \hat{\mu}_{B}\right)}=\hat{\mu}_{B} r_{12}^{B, 1}+\hat{\mu}_{B}^{3} r_{12}^{B, 3}+\ldots
$$



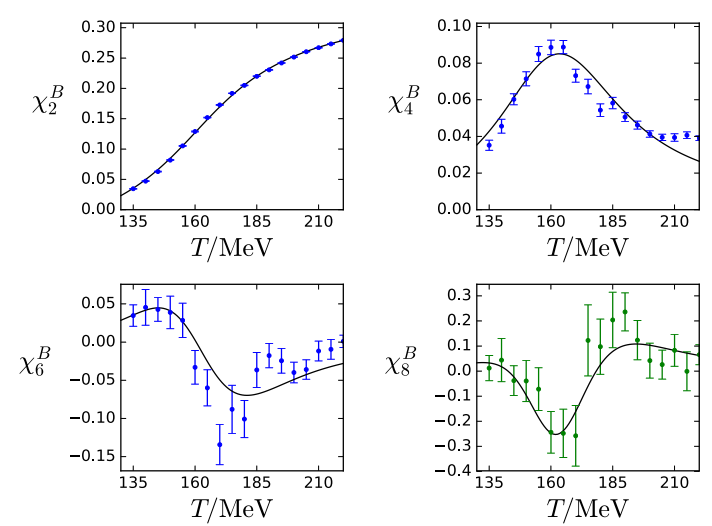

Figure 1: Results for $\chi_{2}^{B}, \chi_{4}^{B}, \chi_{6}^{B}$ and an estimate for $\chi_{8}^{B}$ on a $N_{t}=12$ lattice as functions of the temperature, obtained from the single-temperature analysis (see text). We plot $\chi_{8}^{B}$ in green to point out that its determination is guided by a prior, which is linked to $\chi_{4}^{B}$. The black curve in each panel corresponds to the toy model introduced in $[23,26]$.

$$
\begin{aligned}
& \frac{S_{B} \sigma_{B}^{3}}{M_{B}}=\frac{\chi_{3}^{B}\left(T, \hat{\mu}_{B}\right)}{\chi_{1}^{B}\left(T, \hat{\mu}_{B}\right)}=r_{31}^{B, 0}+\hat{\mu}_{B}^{2} r_{31}^{B, 2}+\ldots \\
& \kappa_{B} \sigma_{B}^{2}=\frac{\chi_{4}^{B}\left(T, \hat{\mu}_{B}\right)}{\chi_{2}^{B}\left(T, \hat{\mu}_{B}\right)}=r_{42}^{B, 0}+\hat{\mu}_{B}^{2} r_{42}^{B, 2}+\ldots
\end{aligned}
$$

The $\mu_{B}$ dependence of the $\chi_{i}^{B}\left(T, \hat{\mu}_{B}\right)$ can be again written in terms of the Taylor expansion:

$$
\begin{aligned}
\chi_{i, j, k}^{B Q S}\left(\hat{\mu}_{B}\right) & =\chi_{i, j, k}^{B Q S}(0)+\hat{\mu}_{B}\left[\chi_{i+1, j, k}^{B Q S}(0)+q_{1} \chi_{i, j+1, k}^{B Q S}(0)+s_{1} \chi_{i, j, k+1}^{B Q S}(0)\right] \\
& +\frac{1}{2} \hat{\mu}_{B}^{2}\left[\chi_{i+2, j, k}^{B Q S}(0)+s_{1}^{2} \chi_{i, j+2, k}^{B Q S}(0)+q_{1}^{2} \chi_{i, j, k+2}^{B Q S}(0)\right. \\
& \left.+2 q_{1} s_{1} \chi_{i, j+1, k+1}^{B Q S}(0)+2 s_{1} \chi_{i+1, j+1, k}^{B Q S}(0)+2 q_{1} \chi_{i+1, j, k+1}^{B Q S}(0)\right]+\ldots
\end{aligned}
$$

with $q_{j}=\frac{1}{j !} \frac{d^{j} \hat{\mu}_{Q}}{\left(d \hat{\mu}_{B}\right)^{j}}(0)$ and $s_{j}=\frac{1}{j !} \frac{d^{j} \hat{\mu}_{S}}{\left(d \hat{\mu}_{B}\right)^{j}}(0)$.

We can now use the constraints $\left\langle n_{S}\right\rangle=0$ and $\left\langle n_{Q}\right\rangle=0.4\left\langle n_{B}\right\rangle$ which can be rewritten as $\chi_{1}^{Q}=0.4 \chi_{1}^{B}$ and $\chi_{1}^{S}=0$ to determine the $r_{i j}^{B, k}$ coefficients form the equations 6,7 and 8 . However we now need to know not only the behavior of the $\chi_{i}^{B}$ but also of derivatives with respect to $\mu_{S}$ and $\mu_{Q}$. For now our simulations are restricted to ensembles with finite $\mu_{B}$. Therefore the $\mu_{S}$ and $\mu_{Q}$ derivatives have to be calculated directly and without the support from the fit that we used in the $\mu_{B}$ direction. We calculate various $\chi_{i, j, k}^{B, Q, S}$ with the appropriate values of $j$ and $k$ and all possible values for $i$ so that $i+j+k \leq 4$. For each group of fluctuations with the same $j$ and $k$ we perform a fit analogous to the procedure described in section 2. This is sufficient to determine the first two $r_{i j}^{B, k}$ coefficients for all three observables. The results are shown in figure 2 . 


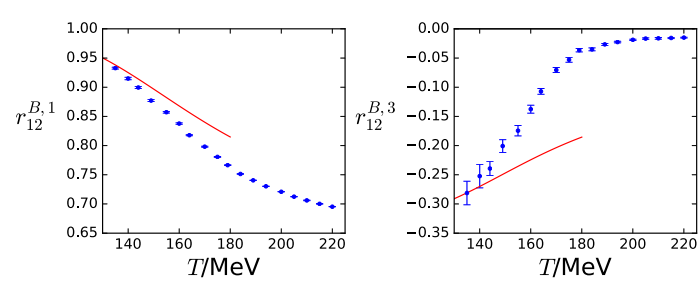

a) $r_{12}^{B, 1}$ (left panel) and $r_{12}^{B, 3}$ (right panel).
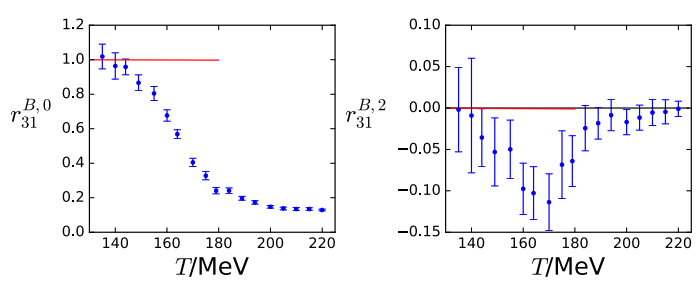

b) $r_{31}^{B, 0}$ (left panel) and $r_{31}^{B, 2}$ (right panel).
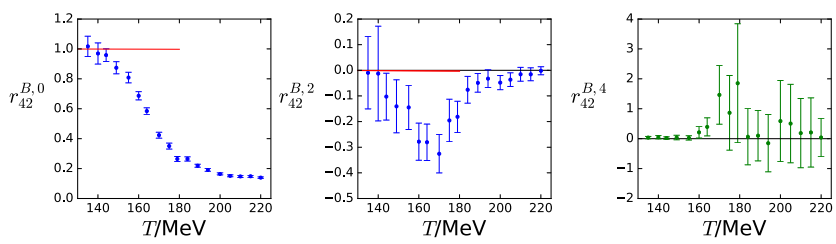

c) $r_{42}^{B, 0}$ (left panel) $r_{42}^{B, 2}$ (middle panel), $r_{42}^{B, 4}$ (right panel). The latter is not obtained independently, but by means of the prior ansatz (see text): for this reason, we plot it in green.

Figure 2: Taylor expansion coefficients as functions of the temperature.

\section{Looking for the critical point}

To look for the critical endpoint in the QCD phase diagram one can try to calculate the radius of convergence of an expansion in $\mu_{B}$. Two obvious expansions for this are either the pressure $p(\mu)=p_{0}+$ $p_{2} \hat{\mu}^{2}+p_{4} \hat{\mu}^{4}+p_{6} \hat{\mu}^{6}+\ldots$ or the fluctuations that are directly related: $\chi_{2}^{B}(\mu)=2 p_{2}+12 p_{4} \hat{\mu}^{2}+30 p_{6} \hat{\mu}^{4}+\ldots$ We define

$$
r_{2 n}^{p}=\sqrt{\frac{p_{2 n}}{p_{2 n+2}}} \text { and } r_{2 n}^{\chi}=\sqrt{\frac{2 n(2 n-1)}{(2 n+1)(2 n+2)}} r_{2 n}^{p} \text {. }
$$

In the limit of $n \rightarrow \infty$ if either $r_{2 n}^{p}$ or $r_{2 n}^{\chi}$ converge, they converge to the same value, the radius of convergence, which guarantees that there is no criticality within this radius. ${ }^{1}$ However, since we only know the fluctuations up to $\chi_{8}^{B}$ as discussed in the previous section, we will first test this procedure for a toy model in which the critical endpoint is known. We use unimproved staggered fermions on an $N_{t}=4$ lattice. For this set up the critical endpoint has been already determined [20,21, 29]. The results for $r_{2 n}^{p}$ and $r_{2 n}^{\chi}$ are shown in the left panel of figure 3. For a temperature where the critical endpoint is close by (right site of the left panel of figure 3), the ratios seem to converge to the correct value. However, as discussed in more detail in ref. [29], due to the structure of $\chi_{6}^{B}$ there is always a temperature for which the ratios seem to converge, independent of the real value for the critical point. For the $N_{t}=12$ data the $r_{2 n}^{\chi}$ and the ratios from the hadron resonance gas are shown in the right panel of figure 3 . Here the errors are still large.

Instead of investigating a toy model with a known critical endpoint, we can also try to describe the data with a toy model without any critical behavior. If one fits the data for $\chi_{1}^{B} / \hat{\mu}_{B}$ at $\mu_{B}=0$ with an

\footnotetext{
${ }^{1}$ As was shown in [28], the ratio estimator is never convergent in a finite volume, and it is problematic even when using the $p_{n}$ extrapolated to infinite volume. It will work if one uses infinite-volume Taylor coefficients and the singularity determining the radius of convergence corresponds to a real phase transition in the infinite-volume limit.
} 

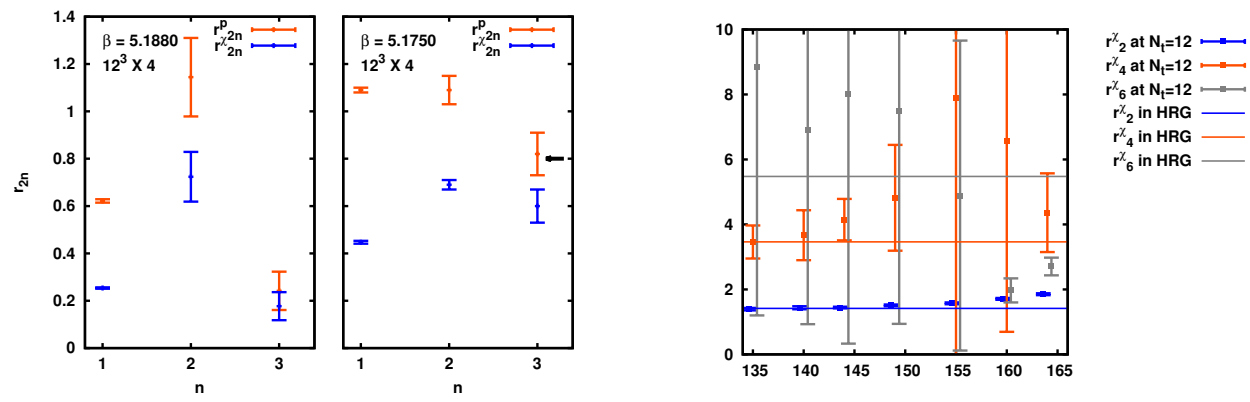

Figure 3: On the left panel: The ratios $r_{2 n}^{p}$ and $r_{2 n}^{\chi}$ (eqn. 10) on an $N_{t}=4$ lattice. On the very left the temperature is close to the crossover temperature. Next to it the temperature is close to the temperature for the critical endpoint. The black arrow marks the value for the critical endpoint from [21]. On the right panel: The $r_{2 n}^{x}$ (eqn. 10) ratios for different temperatures [29].

analytic function of $T$ and assumes that any change with respect to the chemical potential is a linear shift of this function, one can determine all fluctuations analytically (more detail on this toy model can be found in ref. [26]). The results of this toy model are shown with black curves in figure 1 . They agree well with the data.

\section{Acknowledgements}

This project was funded by the DFG grant SFB/TR55. This work was supported by the Hungarian National Research, Development and Innovation Office, NKFIH grants KKP126769 and K113034. An award of computer time was provided by the INCITE program. This research used resources of the Argonne Leadership Computing Facility, which is a DOE Office of Science User Facility supported under Contract DE-AC02-06CH11357. The authors gratefully acknowledge the Gauss Centre for Supercomputing e.V. (www.gauss-centre.eu) for funding this project by providing computing time on the GCS Supercomputer JUQUEEN[30] at Jülich Supercomputing Centre (JSC) as well as on HAZELHEN at HLRS Stuttgart, Germany. This material is based upon work supported by the National Science Foundation under grants no. PHY-1654219 and OAC-1531814 and by the U.S. Department of Energy, Office of Science, Office of Nuclear Physics, within the framework of the Beam Energy Scan Theory (BEST) Topical Collaboration. C.R. also acknowledges the support from the Center of Advanced Computing and Data Systems at the University of Houston. A.P. is supported by the János Bolyai Research Scholarship of the Hungarian Academy of Sciences and by the ÚNKP-19-4 New National Excellence Program of the Ministry for Innovation and Technology. 


\section{References}

[1] Y. Aoki, G. Endrodi, Z. Fodor, S.D. Katz, K.K. Szabo, Nature 443, 675 (2006), hep-lat/0611014

[2] Y. Aoki, S. Borsanyi, S. Durr, Z. Fodor, S.D. Katz, S. Krieg, K.K. Szabo, JHEP 06, 088 (2009), 0903.4155

[3] S. Borsanyi, Z. Fodor, C. Hoelbling, S.D. Katz, S. Krieg, C. Ratti, K.K. Szabo (WuppertalBudapest), JHEP 09, 073 (2010), 1005. 3508

[4] T. Bhattacharya et al., Phys. Rev. Lett. 113, 082001 (2014), 1402 . 5175

[5] A. Bazavov et al., Phys. Rev. D85, 054503 (2012), 1111.1710

[6] C.R. Allton, S. Ejiri, S.J. Hands, O. Kaczmarek, F. Karsch, E. Laermann, C. Schmidt, L. Scorzato, Phys. Rev. D66, 074507 (2002), hep-lat/0204010

[7] C.R. Allton, M. Doring, S. Ejiri, S.J. Hands, O. Kaczmarek, F. Karsch, E. Laermann, K. Redlich, Phys. Rev. D71, 054508 (2005), hep-1at/0501030

[8] R.V. Gavai, S. Gupta, Phys. Rev. D78, 114503 (2008), 0806. 2233

[9] S. Basak et al. (MILC), PoS LATTICE2008, 171 (2008), 0910.0276

[10] O. Kaczmarek, F. Karsch, E. Laermann, C. Miao, S. Mukherjee, P. Petreczky, C. Schmidt, W. Soeldner, W. Unger, Phys. Rev. D83, 014504 (2011), 1011.3130

[11] F. Karsch, Central Eur. J. Phys. 10, 1234 (2012), 1202 . 4173

[12] A. Bazavov et al., Phys. Rev. Lett. 109, 192302 (2012), 1208.1220

[13] S. Borsanyi, Z. Fodor, S.D. Katz, S. Krieg, C. Ratti, K.K. Szabo, Phys. Rev. Lett. 111, 062005 (2013), 1305.5161

[14] S. Borsanyi, Z. Fodor, S.D. Katz, S. Krieg, C. Ratti, K.K. Szabo, Phys. Rev. Lett. 113, 052301 (2014), 1403.4576

[15] M.A. Stephanov, K. Rajagopal, E.V. Shuryak, Phys. Rev. D60, 114028 (1999), hep-ph/9903292

[16] M. Cheng et al., Phys. Rev. D77, 014511 (2008), 0710.0354

[17] Z. Fodor, S.D. Katz, Phys. Lett. B534, 87 (2002), hep-lat/0104001

[18] P. de Forcrand, O. Philipsen, Nucl. Phys. B642, 290 (2002), hep-lat/0205016

[19] M. D’Elia, M.P. Lombardo, Phys. Rev. D67, 014505 (2003), hep-lat/0209146

[20] Z. Fodor, S.D. Katz, JHEP 03, 014 (2002), hep-lat/0106002

[21] Z. Fodor, S.D. Katz, JHEP 04, 050 (2004), hep-lat/0402006

[22] C. Bonati, M. D’Elia, F. Negro, F. Sanfilippo, K. Zambello (2018), 1805.02960

[23] S. Borsanyi, Z. Fodor, J.N. Guenther, S.K. Katz, K.K. Szabo, A. Pasztor, I. Portillo, C. Ratti, JHEP 10, 205 (2018), 1805 . 04445

[24] R. Bellwied, S. Borsanyi, Z. Fodor, S.D. Katz, A. Pasztor, C. Ratti, K.K. Szabo, Phys. Rev. D92, 114505 (2015), 1507.04627

[25] H. Akaike, IEEEE 19, 716 (1974)

[26] S. Borsanyi, Z. Fodor, J.N. Guenther, S.K. Katz, A. Pasztor, I. Portillo, C. Ratti, K.K. Szabó, Nucl. Phys. A982, 223 (2019)

[27] L. Adamczyk et al. (STAR), Phys. Rev. Lett. 112, 032302 (2014), 1309. 5681

[28] M. Giordano, A. Pásztor, Phys. Rev. D99, 114510 (2019), 1904.01974

[29] Z. Fodor, M. Giordano, J.N. Guenther, K. Kapas, S.D. Katz, A. Pasztor, I. Portillo, C. Ratti, D. Sexty, K.K. Szabo (2018), 1807.09862

[30] Jülich Supercomputing Centre, Journal of large-scale research facilities A1, 1 (2015) 